\title{
Comparison of Two Methods to Measure Permeability of Dentin
}

\author{
Jose C. De La Macorra, Nuria I. Escribano \\ Department of Restorative Dentistry, Faculty of Odontology, Complutense University, 28040 Madrid, Spain
}

Received 18 July 2001; revised 1 February 2002; accepted 5 February 2002

\begin{abstract}
Dentin permeability was measured alternatively with two methods: a $10-\mu$ l capillary method with visual evaluation (PC) and a motorized automatic measuring device (Flodec, FD), both interposed in a simulated perfusion system. Eight human third molar coronal fragments were connected to systems, and their permeability to distilled water measured at $0,5,10,15,20,25$, and $29 \mathrm{~cm} \mathrm{H}_{2} \mathrm{O}$ pressure. Resultant permeabilities (in $\mu \mathrm{l} / \mathrm{s}$ ) for both techniques were interrelated with the use of the Passing and Bablok nonparametric method, which gives information about the range of constant and proportional errors and their $95 \%$ confidence intervals $(95 \mathrm{CI})$. The relationship between the methods is described by the regression formula: $\mathrm{FD}=-\mathbf{0 . 0 0 0 3}+\mathbf{0 . 9 4 5} \cdot \mathrm{PC}$, with 95CI for constant $(-0.0015-0.0009)$ and for slope (0.738-1.168), indicating that both methods are interchangeable, although not identical. () 2002 Wiley Periodicals, Inc. J Biomed Mater Res (Appl Biomater) 63: 531-534, 2002
\end{abstract}

Keywords: dentin perfusion; permeability; FLODEC; simulated perfusion; methodology

\section{INTRODUCTION}

Measurements of changes in permeability or of fluid flow through dentin are frequently used for testing sealing ability of restorative adhesive ${ }^{1-5}$ or nonadhesive ${ }^{6}$ materials, mobility of potentially toxic materials, ${ }^{7-9}$ effectiveness of toothpastes ${ }^{10}$ or desensitizing materials, ${ }^{11-14}$ uptake of substances, ${ }^{15}$ or effect of diverse clinical procedures. ${ }^{16-20}$

When the model was first described ${ }^{21}$ the measuring device consisted of a calibrated micropipette of appropriate volume, with an air bubble placed into it, connected via tubes to the specimen on one side and the liquid reservoir on the other. Displacement of the bubble along the micropipette, usually toward the specimen, assessed the amount of fluid movements.

This system is fairly simple, effective, and cheap, but has some inconveniences. First, it does not permit continuous registering of fluid flow. This would be desirable, as in some instances changes must be related to time, and discontinuous registering makes it difficult to accurately distinguish different parts of the recording. Secondly, if changes are to be introduced in the environment via simulation of stimuli to the specimen, recording of the precise timing is fundamental.

Another way of registering fluid flow is by means of an automatic device (FLODEC, de Marco Engineering, Switzerland), designed to keep track of the position of a meniscus

Correspondence to: Jose C. De La Macorra, Dept. Restorative Dentistry, Faculty of Odontology, Complutense University, 28040 Madrid, Spain (e-mail: macorra@ odon.ucm.es)

Contract grant sponsor: Complutense University; contract grant number: BE 314/96 (C) 2002 Wiley Periodicals, Inc. inside a capillary tube. Detection is achieved with an optical system placed outside the tube, which is driven with an electromechanical system to follow the position of the meniscus constantly. Due to the design of the capillary and the coupled electromechanical positioning system, the device is able to detect minor (around 10 to $20 \mathrm{nl}$ ) changes of fluid volume, an important feature in measuring slight variations in specimen flux.

Although this system has been used recently ${ }^{1,7,11,12,22}$ there is no information about the concordance of its measurements with the classic micropipette method. Precision (how close repeated measurements of same quantity are to each other $^{23}$ ) of both methods can be different because, although both are based on similar principles, accuracy of readings can well be different. It has to be kept in mind that in the micropipette method, readings are finally made visually. This makes this method more susceptible to personal determinate errors, ${ }^{23}$ a conscious or subconscious way of biasing readings. For instance readings of position of the air bubble placed between contiguous calibration marks could be consistently assigned to the next higher (or lower) calibration.

If any two methods $(X, Y)$ were always to produce exactly the same results, the relation between would be given by the identity function $Y=X$. But, when both are not identical, the relationship can be modeled by, among others, the model $Y=$ $\alpha+\beta \cdot X+\epsilon$. This linear approach seems to be the best, because it is based in the assumption that both are linearly related (when $Y$ changes, $X$ would change in the same direction and on the same extent). Both variables $(X$ and $Y)$ are interchangeable (it would be the same to test $Y=\alpha+\beta \cdot X+$ $\epsilon \varepsilon$ than to test $X=\alpha+\beta \cdot Y+\epsilon$ ), but are not correlated. Instead, both have the same cause (collateral relation) be- 
cause both measure the same parameter, but measurements of one do not influence the measurements in the other, but through collaterallity. In this model, when $\alpha \neq 0$, there is a constant difference between both methods, and $\alpha$ represents the constant systematic error. On the other hand, when $\beta \neq 1$, both methods differ proportionally and $\beta$ represents the proportional systematic error. Finally, $\epsilon$ is a random, nonsystematic variable representing the random error of both methods. Its mean is equal to 0 . When $\alpha=0$ and $\beta=1$, both methods are interchangeable.

Passing and Bablok regression ${ }^{24,25}$ is a method to estimate intercept $\alpha$, and slope $\beta$, and their $95 \%$ confidence intervals (95CI) nonparametrically. Confidence intervals are used to determine whether there is only a chance difference between $\beta$ and 1 and between $\alpha$ and 0 .

Bland and Altman ${ }^{26}$ proposed one graphical procedure, based on the study of the differences of the results of measuring with two analytical methods. The difference between the results of the two techniques is plotted against the average of both. This helps to identify systematic biases and possible outliers, but is based in some assumptions, namely, that differences between both methods are normally distributed and that there is an independence between the magnitude of differences between methods and the results themselves. These assumptions may be not true, and this method only renders information about the confidence interval of agreement.

The intraclass correlation coefficient (ICC) ${ }^{27,25}$ concept is similar to that of the well-known kappa concordance index for categorical variables, but is applied when data are collected quantitatively. Its main inconveniences are that the rules of interpretation of the value of the ICC are not fully objective, and that it does not differentiate constant from proportional error.

\section{OBJECTIVES}

The objective is to compare, with the use of the Passing and Bablok regression method, accuracy between results of dentin permeability obtained with a micropipette and the FLODEC device.

\section{Materials and Methods}

Eight recently extracted human third molars were used in this study. Specimens were preserved in a $70 \%$ ethanol solution and used within a month after extraction. Occlusal enamel and the roots at furca level were removed with the use of a diamond bur under abundant water refrigeration, exposing the occlusal dentin and the pulpal chamber. The chamber contents were carefully extracted with cotton pliers. Both cutted surfaces were lightly polished with 1200-grit sandpaper (SiC-Paper, Struers A/S, Copenhagen, Denmark) in a water-cooled polishing machine (Struers Dap-7, Struers, Copenhagen, Denmark). The cervical part of the crown segment was glued to a $1 \times 1-\mathrm{cm}$ methacrylate (mma, in Figure 1$)$

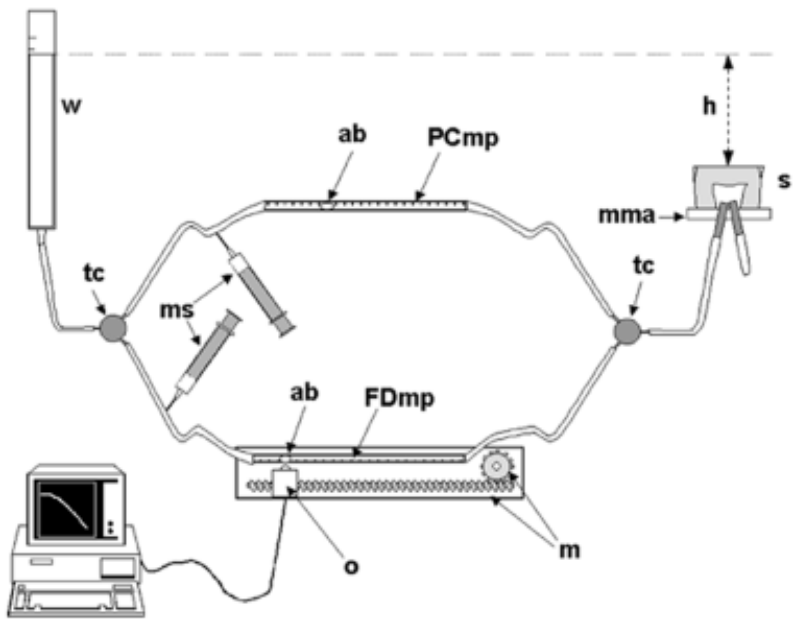

Figure 1. Experimental device diagram. w: water column, tc: threeway tubing connector, ms: microsyringe, ab: air bubble, PCmp: PC method micropipette, FDmp: Flodec device micropipette, o: optical detection, m: motorization of FD device, mma: methyl methacrylate base, s: specimen, h: height of the column of water.

base with a cyanoacrylate general-purpose adhesive (Super Glue 3, Loctite, Madrid, Spain). This base had two holes through which two metal catheters were fitted and sealed (Duralay, Reliance, Dental Mfg. Co., Worth, IL) entering the pulpal chamber.

The oclusal exposed dentin was conditioned with $37 \%$ ortophosphoric acid (Total Etch, batch B28357, Ivoclar/Vivadent, Schaan, Liechtenstein) for $15 \mathrm{~s}$ to eliminate the smear layer created with the diamond bur and washed thoroughly; then the excess water was eliminated with laboratory absorbent paper.

The specimen was connected to a perfusion system, a pressure column of distilled water (w in Figure 1), with a tube through one of the metal catheters. Via the other catheter, air was extracted from the pulpal chamber until it was filled with distilled water. Then the catheter was sealed. The distilled water from the column could get into the specimen through two different plastic tubes joined via a three-way tubing connector (TC-20/3, Small Parts Inc., Miami Lakes, FL), to the opened metal catheter. In one of them, a 10- $\mu$ l micropipette (Microcaps, Drummond Scientific Co., Broomall, PA) was interposed between pressure column and specimen (PCmp in Figure 1). In the other one, an electronic device (Flodec, De Marco Engineering, Geneva, Switzerland) with a capillary tube (FDmp in Figure 1) was interposed between column and crown segment. With microsyringes, air bubbles were introduced in the micropipette and the capillary tube. Along both tubes clamps were placed to stop fluid flow through them alternatively.

All specimens were connected to the perfusion system 24 hours prior to measurements to assure a complete rehydration of the dentinal tissues. Permeability measurements were then carried out. Each specimen was perfused with the use of seven different pressures: 0, 5, 10, 15, 20, 25, and $29 \mathrm{~cm} \mathrm{H}_{2} \mathrm{O}$, for $15 \mathrm{~min}$ per pressure. Six-ten pairs of data were registered 


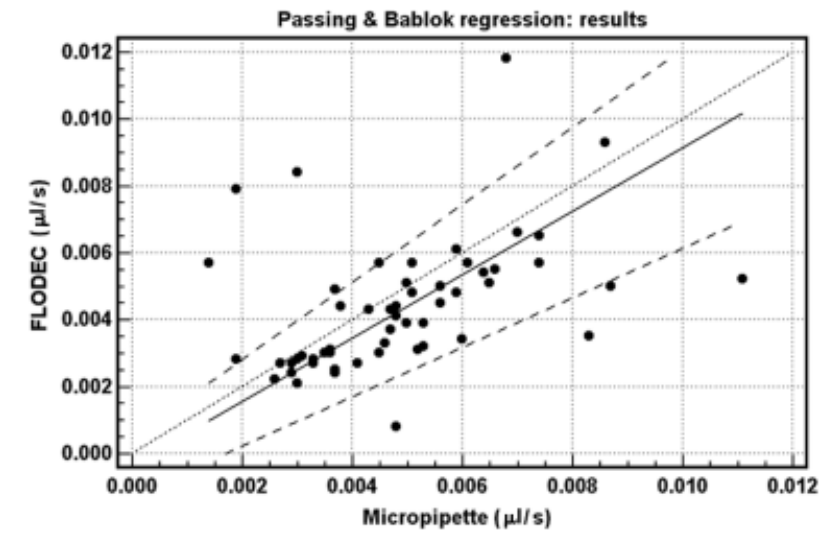

Figure 2. Passing and Bablok regression results. FD: FLODEC device, PC : micropipette, solid line: linear regression, dashed line: $95 \%$ confidence intervals of regression line, dotted line: identity (ideal, i.e.: $\mathrm{PC}=\mathrm{FD})$.

per dentin slice and pressure (PC method). With each pressure, permeability was measured by the two systems, micropipette and FLODEC, alternatively.

Data collected were volume (in $\mu \mathrm{l}$ ) and time (s) for all specimens. Regression through the origin was calculated (SPSS for Windows 9.0.1. SPSS Inc, Chicago, IL) entering time as the independent and volume as the dependent variable, in the formula Volume $(\mu \mathrm{l})=A \cdot$ Time (s). In this way, each specimen had two measures for the slope of the regression line time/vol: the one obtained with the micropipette (PC) and the one obtained with the FLODEC device (FD).

\section{Passing and Bablok Regression}

The problem was formulated under the assumption that the better-known measuring system was the one based in the micropipette (PC). Although not a gold standard, this system has been used for a longer time, and most knowledge on dentin permeability and its changes is based on it. In this way, regression was calculated and CUSUM test for linearity was employed (MedCalc 5.00.13 for Windows, MedCalc Software, Belgium), with PC used as the independent variable and $\mathrm{FC}$ as the dependent one.

\section{RESULTS}

The resulting equation for Passing and Bablok regression was: FD $=-0.0003+0.945 \cdot \mathrm{PC}$ (Figure 2). $95 \mathrm{CI}$ for constant was -0.0015 to 0.0009 , and for the slope was 0.738 to 1.168. The CUSUM test for linearity showed no significant deviation from linearity $(P>0.10)$. Residuals are plotted (Figure 3) against $\mathrm{PC}$ values. This distribution has a regression line (residual $=0.0026-0.518 \cdot \mathrm{PC}$ ), with a Pearson's $r$ coefficient $=0.484, p=0.00016)$ and a slope $(-0.518)$ $(p=0.0002)$ significantly different from 0 (SPSS 9.0, SPSS Inc.).

\section{DISCUSSION}

Election of variables to calculate Passing and Bablok regression (PC as independent and FD as dependent) was arbitrary. This election does not assume that PC values are necessarily more acceptable than FD ones. In fact, if the reverse calculation is made, constant and slope values are the same as if they were calculated with the first formula $(\mathrm{PC}=0.003+$ $1.058 \cdot \mathrm{FD})$. However, it cannot be assumed that either method is more credible than the other.

Constant value is -0.0003 . This is absolutely close to 0 , meaning that it is not probable to have statistically significant constant differences between methods. This is confirmed by observing its $95 \mathrm{CI}(-0.0015$ to 0.0009$)$. This interval contains the value of 0 , meaning that only in $5 \%$ of cases the difference between methods will be significantly different from 0 . This does not mean there are not constant differences at all. There is a certain underestimation of FD method: always, its results are on average slightly lower than results generated with PC method. This is optically shown through the vertical distance (Figure 2) between identity and real regression lines.

Slope value is 0.945 . Its $95 \mathrm{CI}$ is $0.738-1.168$. This interval contains the value of 1 , meaning that only in $5 \%$ of cases will it be significantly different from 1 . Thus, one would not expect to have statistically significant proportional differences between methods, although differences exist, optically shown (Figure 2) through the slightly different slope of (ideal) identity and real regression lines.

CUSUM test for linearity tests whether the distribution departs (or not) significantly from a straight line. In this case it does not $(\mathrm{p}>0.10)$. The above results suggest that both methods are interchangeable.

Residuals are, for each specimen, the difference between the observed value of the dependent variable (FD) and the value predicted by the model. Ideal models will have residuals closer to 0 . Also, better models produce residuals that are evenly distributed, without an apparently asymmetrical or systematic arrangement.

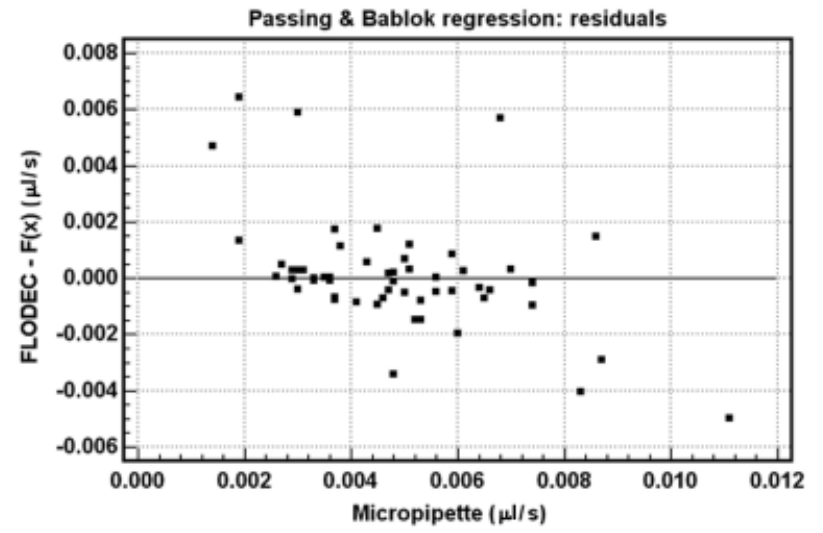

Figure 3. Passing and Bablok regression residuals. FD - F(x): difference between the observed value of the dependent variable (FD) and the value predicted by the model $\mathrm{F}(\mathrm{x}), \mathrm{PC}$ : permeability values with the micropipette method. 
The residuals plot (Figure 3), shows asymmetricall distribution around the 0 . The resiuduals appear to concentrate in the upper-left and lower-right parts. This suggests that, when the result of the PC method is low (a case with low permeability when measured with the micropipette method), the model underestimates value of FD. In other words, for lowest (in our range) PC values, FLODEC device will render data that are to be higher. This is reflected in the slope $(-0.518)$ of the regression line of residuals against PC values. As PC values increase, residuals decrease.

Why do FLODEC device readings tend to be slightly higher than PC ones at low fluid rates and slightly lower than $\mathrm{PC}$ at high fluid rates? One possible explanation may be the difference in the capillarity of the measuring device. The FLODEC device used here has a tube with an internal diameter of $0.68 \mathrm{~mm}$ and, in the present experiment, a capillary of an internal diameter of $0.18 \mathrm{~mm}$ was used as the micropipette in PC method. It is possible that distinct capillary forces within the tubes have an influence on fluid flow.

Another possible explanation is the existence of consistent personal determinate errors ${ }^{23}$ when the micropipette method is used: when fluid rate is lower, the researcher may tend to read minutely lower values and vice versa. But it must be kept in mind that there is not a statistically significant proportional error when FLODEC device is used, compared with PC method. Differences between both methods are small enough to permit them to be considered interchangeable.

\section{CONCLUSION}

Both methods show constant and proportional errors that are statistically negligible.

\section{REFERENCES}

1. Bouillaguet S, Duroux B, Ciucchi B, Sano H. Ability of adhesive systems to seal dentin surfaces: An in vitro study. J Adhesive Dent 2000;2:201-208.

2. Del Nero MO, de la Macorra, JC. Sealing and dentin bond strengths of adhesive systems. Oper Dent 1999; 24:194-202.

3. Gale MS, Darvell, BW. Dentine permeability and tracer tests. J Dent 1999;27:1-11.

4. Pereira PNR, Sano H, Ogata M, Zheng L, Nakajima M, Tagami J, Pashley DH. Effect of region and dentin perfusion on bond strengths of resin-modified glass ionomer cements. J Dent 2000; 28:347-354.

5. Youngson CC, Jones JCG, Fox K, Smith IS, Wood DJ, Gale M. A fluid filtration and clearing technique to assess microleakage associated with three dentine bonding systems. J Dent 1999;27: 223-233.

6. Bachicha WS, DiFiore PM, Miller DA, Lautenschlager EP, Pashley DH. Microleakage of endodontically treated teeth restored with posts. J Endo 1998;24:703-708.

7. Abou Hashieh I, Franquin JC, Cosset A, Dejou J, Camps J. Relationship between dentine hydraulic conductance and the cytotoxicity of four dentine bonding resins in vitro. J Dent 1998;26:473-477.

8. Camps J, Tardieu C, Dejou J, Franquin JC, Ladaique P, Rieu R. In vitro cytotoxicity of dental adhesive systems under simulated pulpal pressure. Dent Mater 1997;13,:34-42.

9. Schmalz G, Schuster U, Nuetzel K, Schweikl H. An in vitro pulp chamber with three-dimensional cell cultures. J Endo 1999;25:24-29.

10. Prati C, Chersoni S, Lucchese A, Pashley DH, Mongiorgi R. Dentin permeability after toothbrushing with different toothpastes. Am J Dent 1999;12:190-193.

11. Bouillaguet S, Virgillito M, Wataha J, Ciucchi B, Holz J. The influence of dentine permeability on cytotoxicity of four dentine bonding systems, in vitro. J Oral Rehab 1998;25:45-51.

12. Camps J, Pizant S, Dejou J, Franquin JC. Effects of desensitizing agents on human dentin permeability. Am J Dent 1998;11: 286-290.

13. Gillam DG, Khan N, Mordan NJ, Barber PM. Scanning electron microscopy (SEM) investigation of selected desensitizing agents in the dentine disc model. End Dent Traumatol 1999;15: 198-204.

14. Zhang Y, Agee K, Pashley DH, Pashley EL. The effects of Pain-Free $\left({ }^{R}\right)$ desensitizer on dentine permeability and tubule occlusion over time, in vitro. J Clin Periodontol 1998;25:884891.

15. Attin T, Schaller HG, Hellwig E. Fluoride uptake in dentin with and without simulating dentinal fluid flow. Clin Oral Invest 1997;1:125-130.

16. Evans CDJ, Wilson PR. The effects of tooth preparation on pressure measured in the pulp chamber: A laboratory study. Int J Prosthet 1999;12:439-443.

17. Lam CW, Wilson PR. The effect of dentine surface treatment on pulpward pressure transmission during crown cementation: A laboratory study. Int Dent J 1998;48:196-202.

18. Moll K, Haller B. Effect of intrinsic and extrinsic moisture on bond strength to dentine. J Oral Rehab 2000;2:149-164.

19. Schaller HG, Weihing T, Strub JR. Permeability of dentine after Nd:YAG laser treatment: An in vitro study. J Oral Rehab 1997;24:274-281.

20. Sekimoto T, Derkson GD, Richardson AS. Effect of cutting instruments on permeability and morphology of the dentin surface. Oper Dent 1999;24:130-136.

21. Derkson GD, Pashley DH, Derkson ME. Microleakage measurement of selected restorative materials: A new in vitro method. J Prosthet Dent 1986;56:435 -440.

22. Ciucchi B, Bouillaguet S, Delaloye M, Holz J. Volume of the internal gap formed under composite restorations in vitro. $\mathrm{J}$ Dent 1997;25: 305-312.

23. Brunette, D.M. Errors of measurement. In: Weikersheimer PB, editor. Critical thinking. Understanding and evaluating dental research. Chicago: Quintessence; 1996. pp 73-81.

24. Passing H, Bablok W. A new biometrical procedure for testing the equality of measurements from two different analytical methods. Application of linear regression procedures for method comparison studies in clinical chemistry. Part I. J Clin Chem Clin Biochem 1983;21:709-720.

25. Domenech JM. Estudio de la concordancia. In: Domenech JM, Granero R, editors. Métodos Estadísticos en Ciencias de la Salud. Barcelona: Signo; 1999. pp 39-58.

26. Bland JM, Altman DG. Statistical method for assessing agreement between two methods of clinical measurement. Lancet 1986;1:307-310.

27. Fleiss JL, Cohen J. The equivalence of weighted kappa and the intraclass correlation coefficient as measures of reliability. Educ Psychol Meas 1973:33;613-619. 